\title{
Spatial transcriptome analysis reveals Notch pathway-associated prognostic markers in IDH1 wild-type glioblastoma involving the subventricular zone
}

Christine Jungk ${ }^{1 *}$, Andreas Mock', Janina Exner ${ }^{1}$, Christoph Geisenberger ${ }^{1}$, Rolf Warta', David Capper ${ }^{2}$, Amir Abdollahi ${ }^{3}$, Sara Friauf ${ }^{1}$, Bernd Lahrmann ${ }^{4}$, Niels Grabe ${ }^{4}$, Philipp Beckhove ${ }^{5,6}$, Andreas von Deimling ${ }^{2}$, Andreas Unterberg ${ }^{1}$ and Christel Herold-Mende ${ }^{1}$

\begin{abstract}
Background: The spatial relationship of glioblastoma (GBM) to the subventricular zone (SVZ) is associated with inferior patient survival. However, the underlying molecular phenotype is largely unknown. We interrogated an SVZ-dependent transcriptome and potential location-specific prognostic markers.

Methods: mRNA microarray data of a discovery set ( $n=36$ GBMs) were analyzed for SVZ-dependent gene expression and process networks using the MetaCore ${ }^{T M}$ workflow. Differential gene expression was confirmed by qPCR in a validation set of 142 IDH1 wild-type GBMs that was also used for survival analysis.
\end{abstract}

Results: Microarray analysis revealed a transcriptome distinctive of SVZ+ GBM that was enriched for genes associated with Notch signaling. No overlap was found to The Cancer Genome Atlas's molecular subtypes. Independent validation of SVZ-dependent expression confirmed four genes with simultaneous prognostic impact: overexpression of HES4 ( $p=0.034 ; \mathrm{HR} 1.55)$ and DLL3 ( $p=0.017 ;$ HR 1.61) predicted inferior, and overexpression of NTRK2 ( $p=0.049 ;$ HR 0.66) and PIR ( $p=0.025 ;$ HR 0.62) superior overall survival (OS). Additionally, overexpression of DLL3 was predictive of shorter progression-free survival (PFS) ( $p=0.043$; HR 1.64). Multivariate analysis revealed overexpression of HES4 to be independently associated with inferior OS ( $p=0.033$; HR 2.03), and overexpression of DLL3 with inferior PFS ( $p=0.046$; HR 1.65).

Conclusions: We identified four genes with SVZ-dependent expression and prognostic significance, among those HES4 and DLL3 as part of Notch signaling, suggesting further evaluation of location-tailored targeted therapies.

Keywords: Glioblastoma, Subventricular zone, mRNA microarray analysis, Location-dependent prognostic markers, Notch signaling

\section{Background}

Despite recent advances in multimodal treatment, de novo glioblastoma (GBM) World Health Organization (WHO) grade IV remains one of the most intractable human cancers, with a median survival of less than 15 months [1] and few long-time survivors [2]. Extensive

\footnotetext{
* Correspondence: christine.jungk@med.uni-heidelberg.de

'Division of Experimental Neurosurgery, Department of Neurosurgery, Ruprecht-Karls-University Heidelberg, Im Neuenheimer Feld 400, 69120 Heidelberg, Germany

Full list of author information is available at the end of the article
}

efforts have been made to maximize the extent of resection (EOR) with simultaneous preservation of neurologic function and quality of life $[3,4]$. At the same time, there are apparent advances in postoperative radiotherapy, chemotherapy, antiangiogenic therapy, immunotherapy, and targeted therapies [5, 6]. Nevertheless, recurrence occurs almost inevitably, in most cases adjacent to the resection cavity, leading to non-standardized salvage therapies and ultimately to death. Treatment failure has been attributed in part to the fact that GBM is not a monoclonal disease but is characterized by intra- and intertumoral 
heterogeneity, resulting in divergent clinical presentation and response to treatment. In this context, several molecular subtypes have been identified $[7,8]$ with distinct driver mutations, prognostic impact, and prediction of treatment response, including a glioma-CpG island methylator phenotype (G-CIMP) [9] which is highly dependent on the presence of mutations in the isocitrate dehydrogenase 1 (IDH1) gene and is associated with improved patient outcome [10]. Tumor location with respect to distinct brain regions reflects another important aspect of intertumoral heterogeneity. In particular, vicinity of de novo GBM to the subventricular zone (SVZ) lining the lateral ventricles, one of the persisting neurogenic regions in the adult human brain $[11,12]$, has been linked to inferior patient outcome [13] and a distinct growth pattern. Lim et al. initially reported a series of 53 de novo GBMs that were preoperatively classified by their vicinity to the SVZ into four groups. Group I consisted of GBMs with the contrast-enhancing (CE) lesion contacting the SVZ and infiltrating the cortex, group II of tumors contacting the SVZ but not involving the cortex, group III of GBMs not contacting the SVZ but involving the cortex, and group IV of tumors neither contacting the SVZ nor infiltrating the cortex [14]. Group I GBMs were most likely to be multifocal at first diagnosis and to recur distant from the resection cavity, while group IV GBMs were always solitary lesions with recurrences exclusively adjacent to the primary site. The authors concluded that GBMs with (SVZ+) and without (SVZ-) contact to the SVZ might arise from different cells of origin and that SVZ+ GBMs might reflect tumors with a high content of SVZ stem cells that have undergone malignant transformation, a hypothesis that has rarely been explored in detail. In one of the few studies addressing this issue so far, Kappadakunnel et al. failed to identify a stem cellderived gene signature by means of a DNA microarray analysis of 47 GBMs classified according to their relationship to the SVZ [15]. In contrast, in a phylogenetic approach making use of intraoperative fluorescence-guided multiple sampling (FGMS) of human GBMs and their adjacent (fluorescent) SVZs, Piccirillo et al. were able to identify the SVZ as a reservoir of malignant precursor clones in the majority of tumors analyzed [16]. Accordingly, several clinical studies have provided evidence that targeting the ipsilateral SVZ by irradiation is associated with superior survival in patients with GBM $[17,18]$, especially in combination with gross total resection (GTR) [19], supporting the hypothesis that the SVZ plays a role in GBM formation and propagation.

Even though SVZ+ GBMs appear to be associated with a distinct clinical and radiographic behavior, little is known about the molecular phenotype underlying these characteristics and potential biomarkers linked to this particular tumor location. Therefore, the aim of this study was to identify a gene signature distinctive of de novo GBM in vicinity to the SVZ and to discover location-dependent genes with a potential prognostic impact. Noteworthy, validation of differential gene expression and prognostic relevance was performed in a confirmatory patient cohort restricted to $I D H$ wild-type $(w t)$ GBM, excluding the unique molecular and prognostic phenotype of $I D H$ mutant $(m t)$ GBM.

\section{Methods \\ Clinical data}

All demographic, treatment-related, and outcome data for patients with de novo GBM treated at the Department of Neurosurgery (University Hospital, Heidelberg, Germany) between 1998 and 2011 were obtained through review of medical charts and gathered in our institutional database. Approval from the ethics committee and written informed consent from patients were obtained in all cases and in accordance with the Declaration of Helsinki. EOR was determined for each patient on magnetic resonance imaging (MRI) scans taken within 72 hours post surgery and was deemed GTR if no residual contrast enhancement was detected; otherwise, EOR was classified as "subtotal" or "unknown" if no postoperative MRI was available. Radiographic classification of GBMs according to their vicinity to the SVZ was performed on preoperative contrastenhanced T1-weighed MR images as described by Lim et al. [14] and depicted in Fig. 1. Group I consisted of tumors contacting the SVZ and infiltrating the cortex, group II of tumors contacting the SVZ only, group III of tumors contacting the cortex only, and group IV of tumors contacting neither SVZ nor cortex. Consequently, groups I and II tumors were pooled as SVZ+ GBM, and groups III and IV tumors as SVZ- GBM.

\section{Microarray cohort}

For microarray analysis, 36 patients with de novo GBM, typical radiographic presentation according to the classification proposed by Lim et al. [14], and availability of high-quality RNA (as described below) were investigated (group I: $n=11$, group II: $n=6$, group III: $n=10$, group IV: $n=9$ ). Median age at first diagnosis was 65 years; median overall survival (OS) and progression-free survival (PFS) were 11 and 3.5 months, respectively. All patients had died by July 2014. The rate of GTR was $31 \% . O^{6}$-methylguanine-DNA methyltransferase (MGMT) promoter hypermethylation was detected in $56 \%$ of patients. IDH1 mutation was present in one patient only (group III). There was no statistical difference regarding age distribution, EOR, survival, and molecular characteristics either between the four groups or when SVZ+ and SVZ- GBMs were compared (Table 1). 


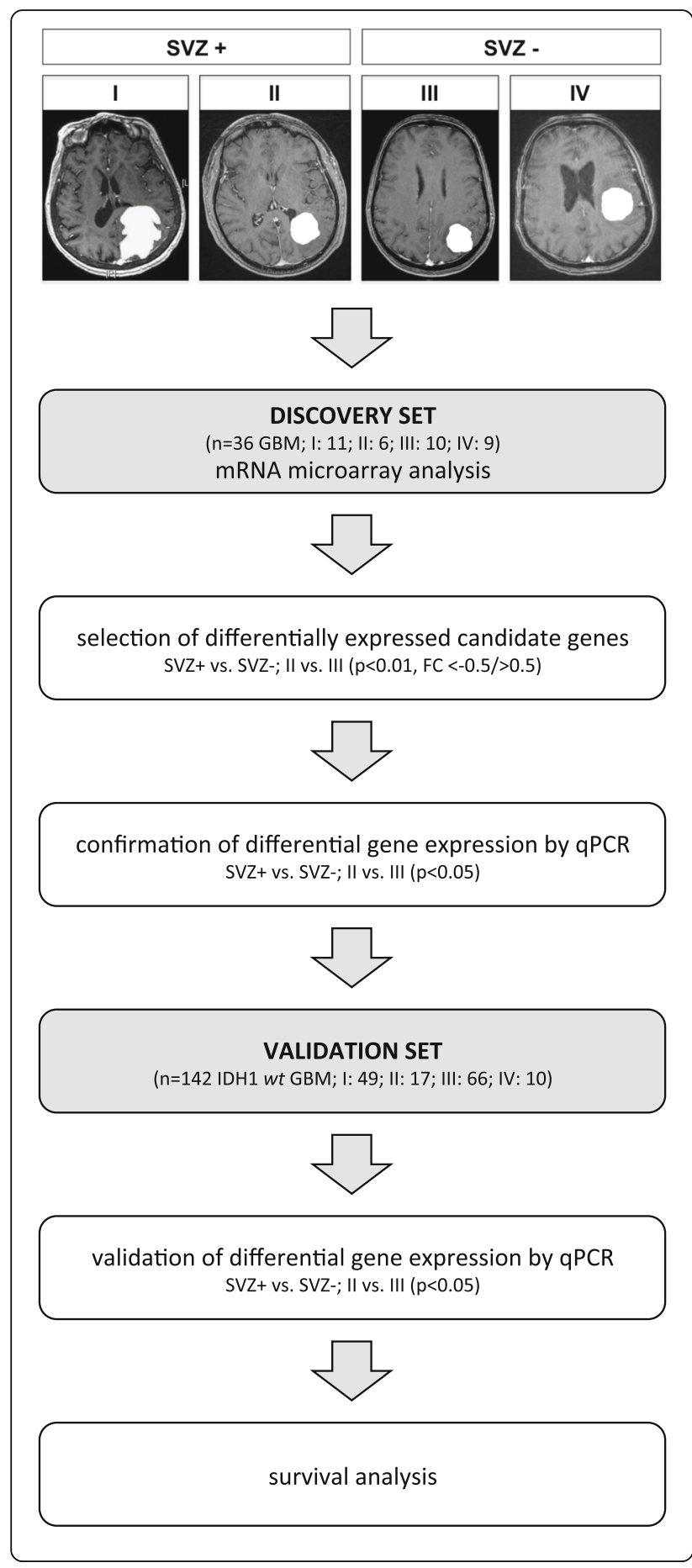

Fig. 1 Flowchart illustrating the experimental design. De novo glioblastomas were allocated to different radiographic groups according to their vicinity to the SVZ as proposed by Lim et al. [14]. Group I consisted of contrast-enhancing tumors contacting the SVZ and infiltrating the cortex, group II of tumors contacting the SVZ only, group III of tumors contacting the cortex only, and group IV of tumors contacting neither SVZ nor cortex. Accordingly, groups I and II tumors were pooled as SVZ+ GBM, groups III and IV tumors as SVZ- GBM. Location-dependent differential gene expression was investigated by mRNA microarray analysis (microarray cohort; $n=36$ GBM) and was validated by QPCR in a confirmatory patient sample (validation cohort; $n=142$ IDH1 wt GBM) with subsequent survival analysis by log-rank test and multivariate Cox regression analysis

\section{Validation cohort}

For independent validation of microarray expression data and evaluation of a prognostic significance of single candidate genes, a validation set of 142 patients with IDH1 wt GBM was analyzed (Table 1) for whom radiographic classification was conducted as well. Median age at first diagnosis was 62 years and median preoperative Karnofsky performance score (KPS) was $82 \%$. Of these patients, $31 \%$ received GTR and the majority postoperative radiation therapy (91\%). Temozolomide (TMZ) was administered in two-thirds of patients. Median OS was 13 months and median PFS was 7 months. Seven patients were alive at the end of the study (July 2014) and thus censored for survival analysis. MGMT promoter hypermethylation was present in $26 \%$, absent in $37 \%$, and not available in $37 \%$ of patients.

\section{Patient material, quality control, and RNA extraction}

Tumor tissue was obtained following surgical resection at the Department of Neurosurgery (University Hospital, Heidelberg, Germany), immediately snap-frozen, and stored at $-80{ }^{\circ} \mathrm{C}$ until further processing. Due to the retrospective nature of this study, the exact sampling position with regard to distance to the SVZ was not determinable; tumors were rather allocated to one of the four location groups based on their radiographic appearance. Two board-certified neuropathologists confirmed histopathological diagnosis and quality control regarding tumor content $(>60 \%)$ and necrosis $(<20 \%)$. Comparing the distribution of tumor content between the four location groups did not reveal a significant difference (Additional file 1: Figure S1A). To ensure that differential gene expression in mRNA microarray analysis was not affected by location-specific differences in tumor microenvironment, we applied the ESTIMATE algorithm from Yoshihara et al. [20], as described in detail in Additional file 1: Figure S1B-D and Additional file 2. IDH1 mutation and MGMT promoter methylation status were determined as described elsewhere [2, 21, 22]. RNA was extracted with the AllPrep DNA/RNA/Protein mini kit (Quiagen, Hilden, Germany) according to the 
Table 1 Patient characteristics of the microarray cohort ( $n=36 \mathrm{GBMs}$ ) and the validation cohort ( $n=142$ IDH1 wt GBMs), presented by location-dependent groups I-IV

\begin{tabular}{|c|c|c|c|c|c|c|}
\hline Group & 1 & $\|$ & III & IV & Total & $p$ value \\
\hline \multicolumn{7}{|l|}{ Microarray cohort } \\
\hline Patients, n (\%) & $11(30)$ & $6(17)$ & $10(28)$ & $9(25)$ & 36 & \\
\hline Sex, $n$ (male : female) & $5: 6$ & $6: 0$ & $7: 3$ & $5: 4$ & $23: 13$ & $0.14^{\mathrm{a}}$ \\
\hline Age at diagnosis, mean (years) & 68 & 64 & 59 & 64 & 65 & $0.21^{\mathrm{b}}$ \\
\hline Extent of resection, $n$ (\%) & & & & & & $0.85^{\mathrm{a}}$ \\
\hline - Complete & $3(27)$ & $2(33)$ & $4(40)$ & $2(22)$ & $11(31)$ & \\
\hline - Partial & $4(36.5)$ & $4(67)$ & $5(50)$ & $5(56)$ & $18(50)$ & \\
\hline - Unknown & $4(36.5)$ & 0 & $1(10)$ & $2(22)$ & $7(19)$ & \\
\hline OS, median (months) & 9 & 9.5 & 15 & 16 & 11.5 & $0.98^{c}$ \\
\hline PFS, median (months) & 3 & 5.5 & 3 & 5 & 3.5 & $0.47^{c}$ \\
\hline Multifocal CE lesion, $n(\%)$ & $2(18)$ & $0(0)$ & $1(10)$ & $0(0)$ & $3(8)$ & $0.42^{\mathrm{a}}$ \\
\hline Multifocal FLAIR lesion, $n$ (\%) & $2(18)$ & $0(0)$ & $3(30)$ & $0(0)$ & $5(14)$ & $0.19^{\mathrm{a}}$ \\
\hline MGMT promoter methylation, $n(\%)$ & & & & & & $0.06^{\mathrm{a}}$ \\
\hline - Yes & $9(82)$ & $1(17)$ & $6(60)$ & $4(44)$ & $20(56)$ & \\
\hline$-\mathrm{No}$ & $2(18)$ & $5(83)$ & $4(40)$ & $5(56)$ & $16(44)$ & \\
\hline IDH1 mutation, $n(\%)$ & & & & & & $0.44^{a}$ \\
\hline - Yes & 0 & 0 & $1(10)$ & 0 & $1(3)$ & \\
\hline$-\mathrm{No}$ & $11(100)$ & $6(100)$ & $9(90)$ & $9(100)$ & $35(97)$ & \\
\hline \multicolumn{7}{|l|}{ Validation cohort } \\
\hline Patients, $n(\%)$ & $49(35)$ & $17(12)$ & $66(46)$ & $10(7)$ & 142 & \\
\hline Sex, $n$ (male : female) & $31: 18$ & $9: 8$ & $41: 25$ & $6: 4$ & $87: 55$ & $0.89^{\mathrm{a}}$ \\
\hline Age at diagnosis, mean (years) & 62 & 57 & 62 & 64 & 62 & $0.32^{d}$ \\
\hline KPS preoperative, mean & 78 & 76 & 86 & 86 & 82 & $0.01^{d}$ \\
\hline Extent of resection, $n(\%)$ & & & & & & $0.006^{\mathrm{a}}$ \\
\hline - Complete & $8(16)$ & $6(35)$ & $23(35)$ & $7(70)$ & $44(31)$ & \\
\hline - Partial & $37(76)$ & $9(53)$ & $31(47)$ & $2(20)$ & $79(56)$ & \\
\hline - Unknown & $4(8)$ & $2(12)$ & $12(18)$ & $1(10)$ & $19(13)$ & \\
\hline Radiation therapy, n (\%) & $43(88)$ & $16(94)$ & $62(94)$ & $8(80)$ & $129(91)$ & $0.4^{\mathrm{a}}$ \\
\hline Temozolomide, $n(\%)$ & $30(61)$ & $14(82)$ & $43(65)$ & $8(80)$ & $95(67)$ & $0.33^{\mathrm{a}}$ \\
\hline OS, median (months) & 12 & 14 & 15 & 17 & 13 & $0.004^{c}$ \\
\hline PFS, median (months) & 6 & 8 & 7 & 8 & 7 & $0.27^{c}$ \\
\hline Censored patients, $n$ (\%) & $2(4)$ & $1(6)$ & $4(6)$ & $0(0)$ & $7(5)$ & $0.85^{\mathrm{a}}$ \\
\hline MGMT promoter methylation & & & & & & $0.43^{\mathrm{a}}$ \\
\hline - Yes, $n(\%)$ & $10(20)$ & $7(41)$ & $17(26)$ & $4(40)$ & $38(26)$ & \\
\hline - No, n (\%) & $16(33)$ & $6(35)$ & $27(41)$ & $3(30)$ & $52(37)$ & \\
\hline - Unknown, n (\%) & $23(47)$ & $4(24)$ & $22(33)$ & $3(30)$ & $52(37)$ & \\
\hline IDH1 mutation, $n(\%)$ & & & & & & N/A \\
\hline - No, n (\%) & $49(100)$ & $17(100)$ & $66(100)$ & $10(100)$ & $142(100)$ & \\
\hline
\end{tabular}

OS overall survival, PFS progression-free survival, CE contrast-enhancing, FLAIR fluid attenuated inversion recovery, MGMT $O^{6}$-methylguanine-DNA methyltransferase, $I D H 1$ isocitrate dehydrogenase 1, KPS Karnofsky performance score

${ }^{a} \mathrm{Chi}$-square test

${ }^{\mathrm{b}}$ Kruskal-Wallis test

'Log-rank test

dOne-way ANOVA

N/A not applicable

In bold: $p<0.01$ 
manufacturer's instructions from high-quality tissue samples. Analyte concentration and quality were determined using a Nanodrop 2000 spectrophotometer (Thermo Scientific) and a Bioanalyzer 2100 (Agilent), respectively.

\section{Processing of microarray data}

$1 \mu \mathrm{g}$ total RNA from 36 GBM tissues was submitted to the Genomics Core Facilities of the German Cancer Research Center (DKFZ, Heidelberg, Germany) for microarray analysis. After purification, reverse transcription to cDNA, and labeling according to the Illumina protocol [23], samples were hybridized to Human HT-12 v.4.0 arrays (Illumina). Raw-intensity data were obtained after image analysis of the fluorescent spot intensity reads. All preprocessing and normalization steps were performed in the $R$ programming environment [www.r-project.org]. Interarray normalization was conducted using qspline normalization in the affy package [24, 25]. After median probe set summarization, a linear model was fitted to account for different batches (limma package). Lastly, intraarray normalization was performed by median centering of the data, followed by log2 transformation. Data were deposited at the NCBI Gene Expression Omnibus [GEO:GSE83537].

\section{Assessment of molecular subtypes in microarray cohort}

Centroids established by Verhaak et al. [8] for subtyping of GBM expression data were downloaded from The Cancer Genome Atlas (TCGA) working group website (the accompanying data freeze was released with the aforementioned publication). For each case, correlation (Pearson's $r$ ) between respective expression values and centroids was calculated for all genes available in the data set $(n=800$ out of 840$)$. Subsequently, each sample was assigned the subtype of the centroid with which it was most strongly correlated.

\section{Real-time PCR}

Quantitative PCR (qPCR) was performed to confirm mRNA microarray expression data and differential expression of single candidate genes in the validation cohort. Primer design and selection of corresponding hybridization probes was done using the Universal ProbeLibrary Assay Design Center (http://lifescience.roche.com). Primers were obtained from Sigma-Aldrich (St. Louis, MO, USA) and along with the probes are summarized in Additional file 3: Table S1. RT-PCR reactions were performed according to the manufacturer's instructions using 45 amplification cycles (LightCycler LC480, cDNA First Strand Transcriptor Kit, LightCycler TaqMan Master, Universal ProbeLibrary Set (human); all Roche Diagnostics, Mannheim, Germany). Quantification of mRNA expression was performed in triplicate and referenced to a set of housekeeping genes: glyceraldehyde-3-phosphate dehydrogenase
(GAPDH), beta-actin (ACTB), and hypoxanthine-guanine phosphoribosyltransferase 1 (HPRT1). Only triplicates with a deviation in crossing point (Cp) values of less than $<0.55$ were deemed appropriate for further relative quantification employing qbase + software version 2.5 (Biogazelle NV, Zwijnaarde, Belgium).

\section{Statistical analyses}

Unless stated otherwise, statistical analyses were conducted in $R$ (www.r-project.org). Differential gene expression in GBM subgroups was assessed using a twosided Student's $t$ test and a Mann-Whitney test as indicated. For survival analysis, PFS and OS were used as end points. PFS was defined as the time interval from first histologic diagnosis to radiologic signs of progression/recurrence or death, whatever occurred first. OS was defined as the time interval from first histologic diagnosis until death or last follow-up. Prognostic significance was determined using univariate and multivariate Cox regression analyses and log-rank tests. For multivariate models, all clinico-pathological parameters significant in the univariate analysis were included. Enrichment analysis for process networks was performed using the MetaCore ${ }^{\mathrm{Tw}}$ analysis workflow.

\section{Results}

\section{SVZ-dependent transcriptional profiles in de novo GBM}

First, an mRNA microarray analysis was performed for a discovery set of 36 de novo GBMs (microarray cohort) allocated to one of the four radiographic groups proposed by Lim et al. [14]. Hierarchical clustering of the top 222 genes differentially expressed between SVZ+ and SVZ- GBMs $(p<0.01)$ revealed distinct transcriptional profiles that perfectly discriminated between these two groups (Fig. 2a). This effect was most pronounced when only the two most contrary GBM groups (group II (contacting SVZ only) and group III (contacting cortex only)) that separate best between GBMs with and without SVZ involvement were compared (top 312 genes; $p$ $<0.01$ ) (Fig. 2b). To learn more about the functional significance of these gene signatures, a MetaCore ${ }^{\mathrm{Tm}}$ enrichment analysis was performed to identify the top 10 pathways associated with differential gene expression. Enrichment analysis of the top 1494 genes differentially expressed between SVZ+ and SVZ- GBMs $(p<0.05)$ revealed upregulation of genes linked to chromatin modification and downregulation of genes linked to Notch signaling, blood vessel morphogenesis, and immune modulation ( $\mathrm{T}$ cell receptor signaling, interleukin-2 (IL-2) signaling, leukocyte chemotaxis) in SVZ+ GBMs (Table 2). Comparing the top 1573 genes differentially expressed between group II and group III GBMs $(p<0.05)$, genes related to neurogenesis were upregulated, and genes related to blood vessel morphogenesis and immune modulation 

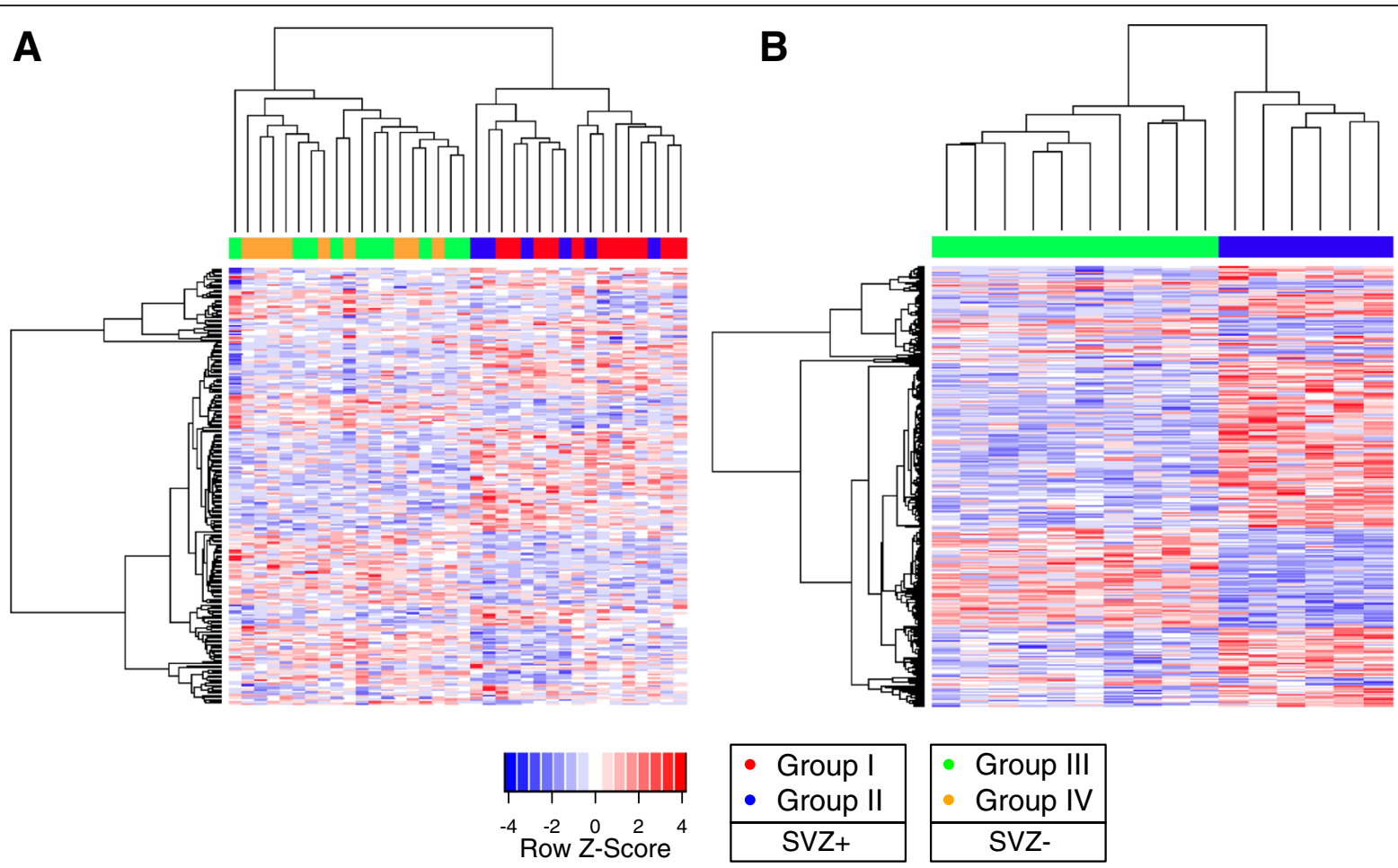

C

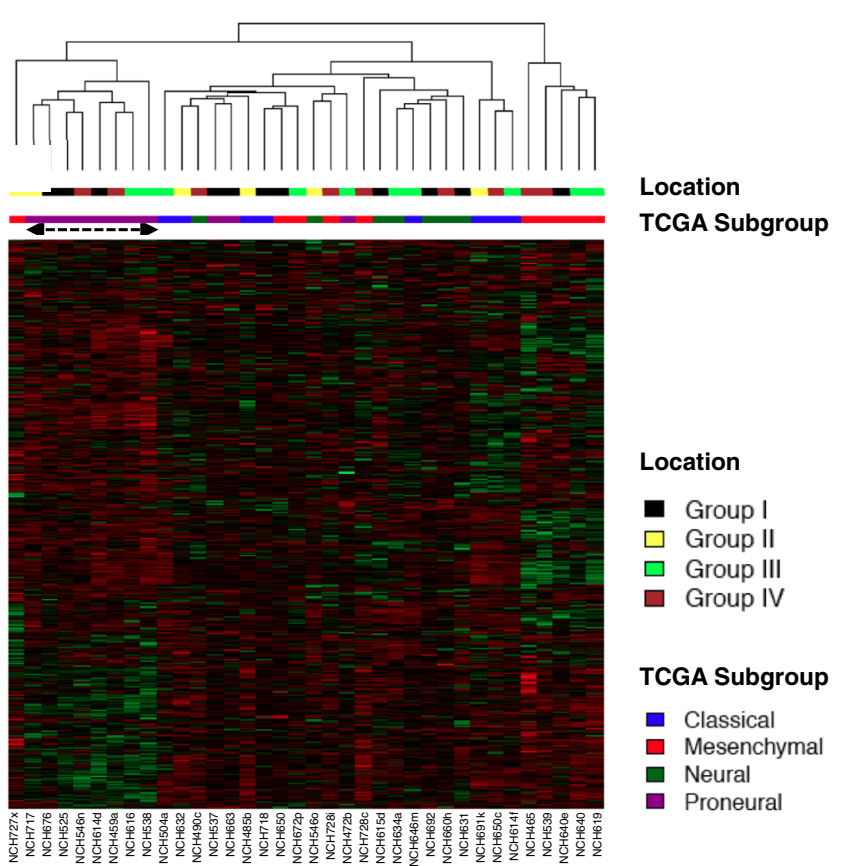

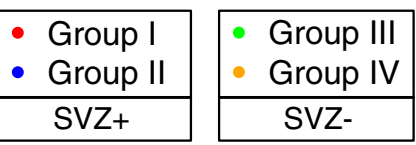

D

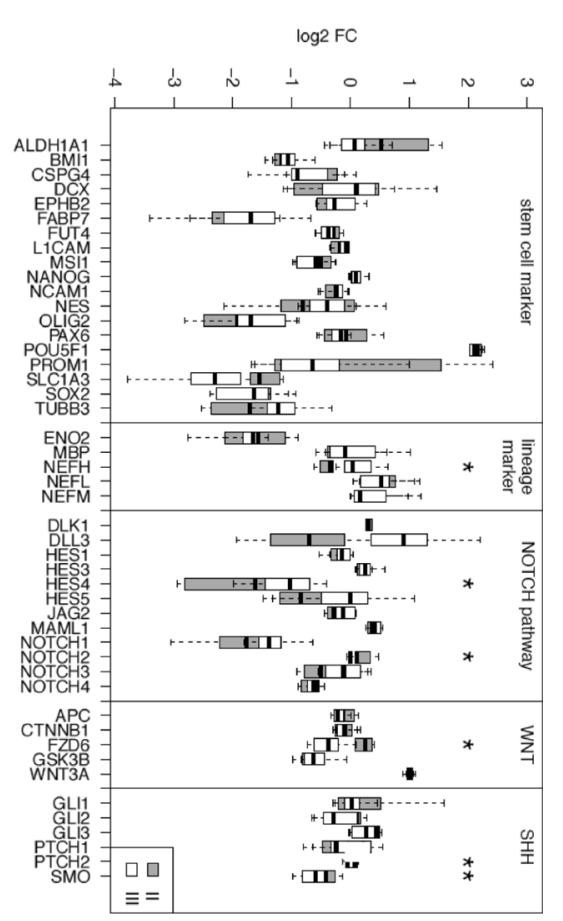

Fig. 2 a, b Hierarchical clustering of mRNA microarray data revealed two main transcriptional profiles that reflect the allocation of tumors according to their vicinity to the SVZ in general (a; top 222 genes differentially expressed between SVZ+ and SVZ- GBMs $(p<0.01)$ ) and to group II (GBMs contacting the SVZ only) and group III (GBMs contacting the cortex only) tumors in particular (b; top 312 genes; $p<0.01$ ). c There was no overlap between location-dependent (SVZ+/-) gene signatures and the molecular subtypes reported by the TCGA working group. $\mathbf{d}$ Box plots depicting differential expression of genes considered as stem cell markers, lineage markers, and markers of three developmental pathways (Notch, Wnt, Sonic Hedgehog (SHH)) as identified by mRNA microarray analysis. Note that significant differences $(p<0.05$; marked with asterisk) were observed for a subset of genes only (FZD6, PTCH2, NOTCH2, HES4, NEFH) together with a strong trend for DLL3 $(p=0.0654)$, notably including three genes with involvement of Notch signaling 
Table 2 Summary of enrichment analysis for the top 1494 differentially expressed genes between groups I, II (SVZ+) versus III, IV $($ SVZ-) $(p<0.05)$

\begin{tabular}{|c|c|c|}
\hline MetaCore ${ }^{\mathrm{TM}}$ process network & Upregulated in SVZ+ $(n=771)$ & Downregulated in SVZ+ $(n=723)$ \\
\hline Term & $p$ value $\left(n_{\text {upregulated }} / n_{\text {total }}\right)$ & $p$ value $\left(n_{\text {downregulated }} / n_{\text {total }}\right)$ \\
\hline Blood vessel morphogenesis & $0.669(4 / 228)$ & $1.55 \mathrm{E}-04(16 / 228)$ \\
\hline Regulation of epithelial-to-mesenchymal transition & $0.943(2 / 226)$ & 4.57E-04 (15/226) \\
\hline Leukocyte chemotaxis & $0.985(1 / 205)$ & 5.32E-04 (14/205) \\
\hline Chromatin modification & $9.61 \mathrm{E}-04(9 / 128)$ & $0.392(4 / 128)$ \\
\hline T cell receptor signaling & $0.971(1 / 174)$ & $1.22 \mathrm{E}-03(12 / 174)$ \\
\hline Cytoskeleton - actin filaments & $0.465(4 / 176)$ & 1.35E-03 (12/176) \\
\hline Notch signaling & $0.507(5 / 236)$ & \multirow{2}{*}{$\begin{array}{l}2.08 \mathrm{E}-03(14 / 236) \\
0.480(4 / 144)\end{array}$} \\
\hline Skeletal muscle development & $2.19 \mathrm{E}-03(9 / 144)$ & \\
\hline Cell cycle G1/S & $0.407(4 / 163)$ & $2.31 \mathrm{E}-03(11 / 163)$ \\
\hline Inflammation - IL-2 signaling & $0.878(1 / 104)$ & 4.11E-03 (8/104) \\
\hline
\end{tabular}

The top 10 enrichments according to $p$ value are depicted (in bold: $p<0.01$ ). In addition, the total number of genes per process network ( $n_{\text {total }}$ as well as the intersect with significantly up- or downregulated genes are displayed ( $n_{\text {upregulated }}$ and $n_{\text {downregulated }}$ )

(IL-2 signaling, leukocyte chemotaxis) were downregulated in group II GBMs contacting the SVZ only (Table 3). We further explored a potential overlap between SVZ-dependent transcriptomes and the molecular subtypes reported by the TCGA working group [8] but found none (Fig. 2c).

Identification of candidate genes distinctive of SVZ+ GBM To identify single candidate genes with locationdependent differential expression, mRNA microarray data were further compared between SVZ+ and SVZ- GBMs and group II and group III GBMs, respectively. Candidate genes had to meet the following criteria: a $p$ value (two-sided $t$ test) of $<0.01$ and/or a $\log 2$ fold change (FC) of $>0.5 /<-0.5$ in at least one of the two comparisons (Fig. 1). Thus, 26 genes were identified (Table 4). To exclude false positive results, $\mathrm{qPCR}$ analysis of
mRNA expression was performed for all tumor samples of the microarray cohort. Differential gene expression on a $p<0.05$ level (two-sided Mann-Whitney test) was verified for 16 genes: PIR (pirin), HES4 (hairy and enhancer of split 4), DLL3 (delta-like 3), NTRK2 (neurotrophic receptor tyrosine kinase type 2), IGFBP5 (insulin-like growth factor-binding protein 5), BAI3 (brain-specific angiogenesis inhibitor 3), EMILIN-3 (elastin microfibril interfacer 3), FERMT2 (fermitin family member 2), $\mathrm{CDH} 4$ (cadherin 4), HIF1A (hypoxia inducible factor 1, alpha subunit), RBP1 (retinol binding protein 1), SYTL4 (synaptotagminlike 4), THBS4 (thrombospondin 4), FZD6 (frizzled class receptor 6), ENPP5 (ectonucleotide pyrophosphatase/ phosphodiesterase 5), and BATF3 (basic leucine zipper transcription factor, ATF-like 3). See Table 4. Two of these genes (EMILIN-3 and CDH4) have never been reported in the context of glioma research so far. BATF3 had to be

Table 3 Summary of enrichment analysis for the top 1573 differentially expressed genes between groups II and III ( $p<0.05)$

\begin{tabular}{lll}
\hline MetaCore & Upregulated in group II $(n=957)$ & $\begin{array}{l}\text { Downregulated in group II (n=616) } \\
\text { Term }\end{array}$ \\
\hline Blood vessel morphogenesis $\left(n_{\text {downregulated }} / n_{\text {total }}\right)$
\end{tabular}

The top 10 enrichments according to $p$ value are depicted (in bold: $p<0.01$ ). In addition, the total number of genes per process network ( $n_{\text {total }}$ ) as well as the intersect with significantly up- or downregulated genes are displayed ( $n_{\text {upregulated }}$ and $n_{\text {downregulated }}$ ) PI3K phosphatidylinositide 3-kinase, Akt protein kinase B 
Table 4 Tabular summary of SVZ-dependent expression of genes

\begin{tabular}{|c|c|c|c|c|c|c|c|c|c|c|}
\hline \multirow[t]{3}{*}{ Gene } & \multicolumn{6}{|c|}{ Microarray cohort $(n=36)$} & \multirow{3}{*}{$\begin{array}{l}\text { Selected } \\
\text { for validation }\end{array}$} & \multicolumn{3}{|c|}{ Validation cohort $(n=142)$} \\
\hline & \multicolumn{3}{|c|}{ SVZ+ vs. SVZ- } & \multicolumn{3}{|l|}{ II vs. III } & & \multirow{2}{*}{$\begin{array}{l}\text { SVZ+ vs. SVZ- } \\
p \text { value }\end{array}$} & \multirow{2}{*}{$\begin{array}{l}\| \text { vs. II } \\
p \text { value }\end{array}$} & \multirow[t]{2}{*}{ Expression in SVZ+/II } \\
\hline & $p$ value & FC & $\mathrm{qPCR}$ & $p$ value & FC & $\mathrm{qPCR}$ & & & & \\
\hline PIR & 0.0188 & 0.4770 & 0.0082 & 0.0047 & 0.7338 & 0.0225 & $\checkmark$ & 0.1597 & 0.05 & Low \\
\hline HES4 & 0.3680 & -0.0135 & 0.0015 & 0.0440 & -0.5945 & 0.5584 & $\checkmark$ & 0.0102 & 0.0007 & High \\
\hline DLL3 & 0.5645 & -0.7725 & 0.1407 & 0.0654 & -1.5897 & 0.0295 & $\checkmark$ & 0.9516 & 0.0857 & High (trend) \\
\hline NTRK2 & 0.0511 & 0.2464 & 0.5821 & 0.0304 & 0.5708 & 0.0559 & $\checkmark$ & 0.7883 & 0.0558 & High (trend) \\
\hline IGFBP5 & 0.0134 & 0.4772 & 0.0066 & 0.0234 & 1.2149 & 0.1179 & $\checkmark$ & 0.8477 & 0.9498 & - \\
\hline$B A / 3$ & 0.3676 & -0.0817 & 0.1829 & 0.0290 & -0.5699 & 0.0714 & $\checkmark$ & 0.2911 & 0.8653 & \\
\hline EMILIN3 & 0.0810 & 0.3924 & 0.3921 & 0.0290 & 0.7203 & 0.0312 & $\checkmark$ & 0.6789 & 0.5567 & \\
\hline FERMT2 & 0.0109 & -0.1693 & 0.0058 & 0.0088 & -0.8139 & 0.0017 & $\checkmark$ & 0.7765 & 0.1156 & \\
\hline $\mathrm{CDH} 4$ & 0.0197 & -0.3874 & 0.0168 & 0.0929 & -0.7704 & 0.0120 & $\checkmark$ & 0.2006 & 0.7516 & \\
\hline UCHLI & 0.0168 & 0.3306 & 0.1369 & 0.2702 & 0.1823 & 0.7063 & & & & \\
\hline HIF1A & 0.0052 & -0.1433 & 0.0215 & 0.1274 & -0.1174 & 0.5345 & $\checkmark$ & 0.6371 & 0.7699 & \\
\hline TGFB3 & 0.0021 & -0.5547 & 0.2056 & 0.0570 & -0.6072 & 0.3000 & & & & \\
\hline$R B P 1$ & 0.0017 & 0.6986 & 0.0186 & 0.0035 & 1.2351 & 0.0312 & $\checkmark$ & 0.9776 & 0.6936 & \\
\hline SYTL4 & 0.1318 & 0.0785 & 0.2347 & 0.0035 & 1.0968 & 0.0075 & $\checkmark$ & 0.3189 & 0.4702 & \\
\hline THBS4 & 0.0155 & 0.1638 & 0.1810 & 0.0096 & 0.8009 & 0.0072 & $\checkmark$ & 0.4495 & 0.9817 & \\
\hline NODAL & 0.0043 & -0.4242 & 0.3117 & 0.0225 & -0.4279 & 0.2625 & & & & \\
\hline FZD6 & 0.1895 & 0.1517 & 0.4073 & 0.0014 & 0.6362 & 0.0730 & & & & \\
\hline VAV3 & 0.0873 & 0.1032 & 0.5665 & 0.0235 & 0.8138 & 0.4895 & & & & \\
\hline ENPP5 & 0.3551 & 0.2665 & 0.0397 & 0.0218 & 0.6382 & 0.0116 & & & & \\
\hline TIMP4 & 0.4696 & 0.1804 & 0.1286 & 0.0417 & 0.5615 & 0.1179 & & & & \\
\hline$N D N$ & 0.0107 & 0.8016 & 0.2038 & 0.2543 & 1.3415 & 0.9458 & & & & \\
\hline NFKBIA & 0.0149 & -0.1440 & 0.3924 & 0.0144 & -0.3358 & 0.2515 & & & & \\
\hline IRF9 & 0.0750 & -0.0766 & 0.2604 & 0.0157 & -0.5397 & 0.2630 & & & & \\
\hline BATF3 & 0.1257 & -0.1263 & 0.0414 & 0.0087 & -0.6015 & 0.6570 & & & & \\
\hline ANGPTL2 & 0.2385 & -0.1070 & 0.5178 & 0.0187 & -0.6711 & 0.7632 & & & & \\
\hline PDGFRA & 0.9394 & -0.0442 & 0.2959 & 0.0453 & -1.1161 & 0.0714 & & & & \\
\hline
\end{tabular}

First, mRNA microarray data were compared between SVZ+ and SVZ- GBM and group II and group III GBM, respectively (in bold: $p<0.01$ (two-sided $t$ test), log2 fold change $(F C)>0.5 /<-0.5$ ) with technical validation of differential gene expression by $q P C R$ (in bold: $p<0.05$ (two-sided Mann-Whitney test)) (microarray cohort). Based on the prespecified significance levels, 13 candidate genes (indicated by check marks) were chosen for validation of differential gene expression by qPCR (in bold: $p<0.05$ (two-sided Mann-Whitney test)) in a confirmatory patient cohort (validation cohort)

excluded from further analysis in the validation cohort due to a negative correlation of expression data, and FZD6 and ENPP5 due to a high variance of expression values. All other genes $(n=13)$ were subjected to an independent validation of differential gene expression by means of qPCR in the validation cohort $(n=142$ patients with IDH1 wt GBM).

As expected, independent validation of locationdependent differential expression was accomplished for a subset of genes only. In SVZ+ GBMs, upregulation of HES4 $(p=0.01)$ was observed, a finding that was even more pronounced $(p=0.0007)$ when group II GBMs (contacting the SVZ only) were compared to group III GBMs (contacting the cortex only). In group II GBMs, there was also a strong trend for upregulation of $D L L 3$ $(p=0.086)$ and NTRK2 $(p=0.056)$ and downregulation of PIR ( $p=0.05)$ (Table 4; Fig. 3a, b).

\section{SVZ-dependent regulation of genes involved in Notch signaling}

It has been hypothesized that de novo GBMs with and without contact to the SVZ are derived from different cells of origin with SVZ+ GBMs enriched for (cancer) stem cells $[11,12,14]$. In this study, MetaCore ${ }^{\mathrm{m} w}$ pathway analysis of mRNA microarray data did not reveal enrichment in classical (cancer) stem cell pathways except for a downregulation of genes linked to Notch signaling in SVZ+ GBMs (Table 2). When comparing the two most contrary location-specific groups II and III by means of MetaCore $^{\text {max}}$, Notch signaling was not among the top 


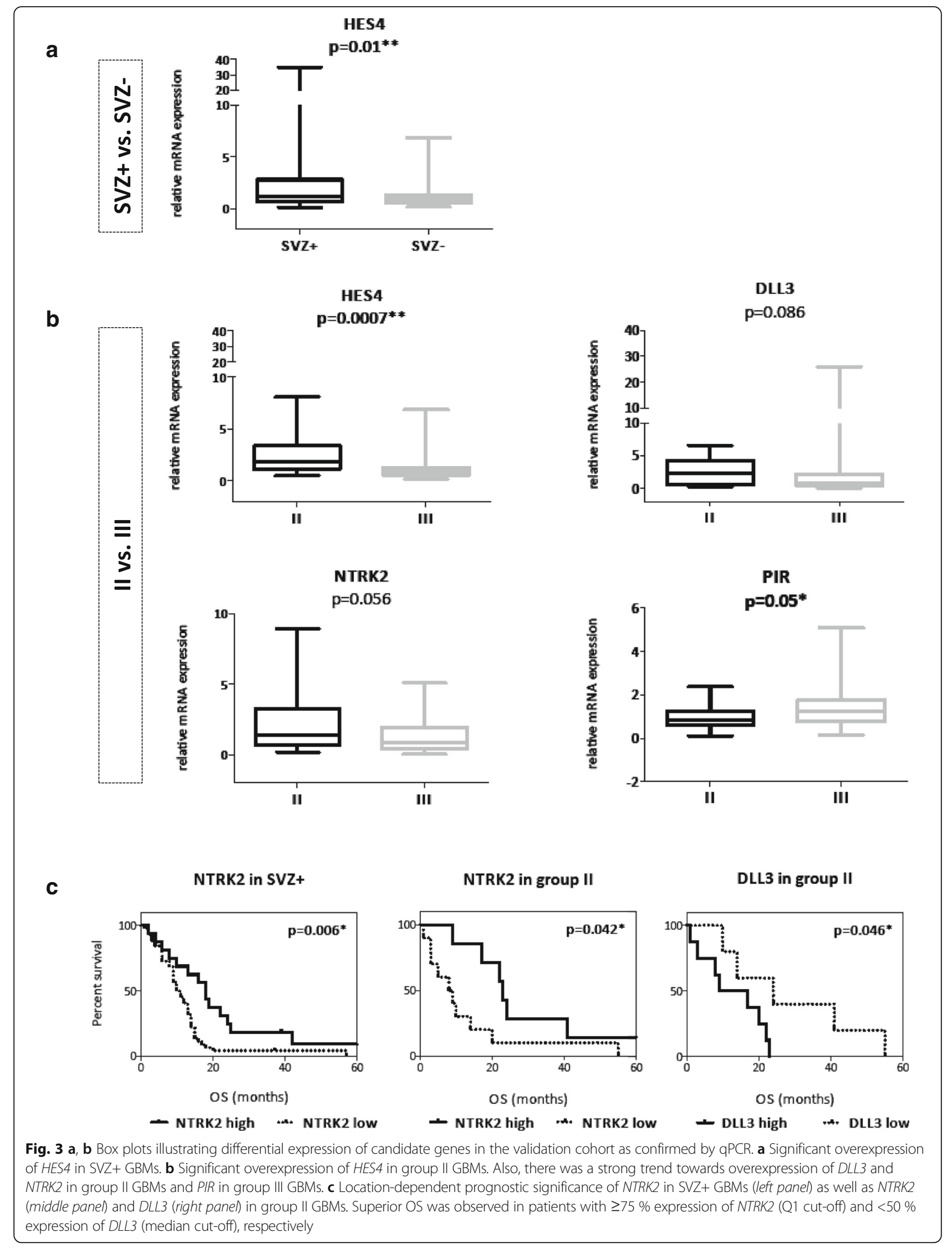


signaling networks, but group II GBMs were enriched for genes related to neurogenesis (Table 3). Keep in mind, however, that a significant $p$ value for the enrichment analysis does not necessarily imply a meaningful down- or upregulation of the pathway, but a mere enrichment of the differentially upregulated or downregulated genes for the respective process network (Tables 2 and 3). As enrichment analyses with a curated gene list or process networks can only serve as a starting point for further analysis, we decided to hand-search our mRNA microarray data for an additional selection of 47 published markers of neural stem cells, radial glia cells, and brain tumor-initiating cells (BTICs), lineage markers and key players of three developmental pathways (Notch, Wnt, Sonic Hedgehog) in view of a potential location-dependent difference in gene expression. In this regard, we chose to compare groups II and III GBMs since this represents the most distinct separation between GBMs with (group II) and without (group III) contact to the SVZ (Fig. 2d). However, an unequivocal SVZ-dependent (cancer) stem cell signature was not detected. In fact, differential gene expression was observed for a subset of genes only (FZD6, NOTCH2, PTCH2 and HES4, DLL3, NEFH, respectively). Notably, three of these genes (DLL3, NOTCH2, HES4) are involved in Notch signaling: $D L L 3$ is a ligand to Notch receptors (among those NOTCH2), while HES4 is a target gene of Notch signaling that acts as a tissue-specific repressor (Fig. 4). This observation prompted us to search our microarray data for further components of the Notch pathway. Indeed, a non-significant differential regulation was found for PSEN2 (presenilin2) and NCSTN (nicastrin), two genes involved in the intracellular cleavage of Notch receptors, and for MAML3 (mastermind like transcriptional coactivator 3) that amplifies Notchinduced transcription (Fig. 4). For HES4 and DLL3, SVZdependent differential gene expression was confirmed in further analyses of the validation cohort (Table 4; Fig. 3a, b), along with a significant prognostic impact on patient outcome (Table 5), as described below.

\section{Identification of prognostic markers distinctive of SVZ+ GBM} In our validation cohort of 142 patients with $I D H w t$ GBM, age at first diagnosis, radiotherapy, adjuvant chemotherapy, preoperative KPS and GTR, together with a clear trend for SVZ involvement $(p=0.056$; HR 1.39 $(0.99-1.96))$, were predictive for OS, while age at first diagnosis and adjuvant chemotherapy were predictive for PFS (Table 5). In the multivariate analysis, vicinity to the SVZ (SVZ+ GBM) was an independent prognosticator of inferior OS ( $p=0.023$; HR 1.82 (1.09-3.04); 12 versus 15 months) but did not affect PFS (Table 5). As expected from the literature, GTR was an independent prognosticator of superior OS $(p=0.007$; HR 0.48
$(0.29-0.83))$. Note that the rate of GTR was significantly higher in SVZ- GBM compared to SVZ+ GBM (39 \% versus $21 \% ; p=0.019$; Fisher's exact test). Radiotherapy ( $p=0.002$; HR $0.22(0.08-0.58))$ and age at first diagnosis $(p=0.024$; HR $1.03(1.00-1.06))$ were also associated with OS in the multivariate analysis.

Next, we asked if our location-specific candidate genes discovered by microarray analysis (HES4, DLL3, NTRK2, $P I R)$ conferred a prognostic impact; therefore, we investigated a possible association between mRNA expression levels and patient outcome in the validation cohort. Since nothing is known about the biological power of candidate gene expression levels, expression data were dichotomized both according to the median in "high" and "low" expression ("median cut-off") and according to quartiles either in "top $25 \%$ expression" and " $<75 \%$ expression" ("Q1 cut-off") or "bottom $25 \%$ expression" and "> $25 \%$ expression" ("Q3 cut-off"). Univariate analysis revealed a prognostic impact for all of these genes. High expression of HES4 ( $p=0.034$, HR 1.55 (1.03-2.32); Q3 cut-off) and DLL3 ( $p=0.017$, HR 1.61 (1.08-2.39); median cut-off) predicted inferior OS, while high expression of NTRK2 ( $p=0.049$, HR 0.66 (0.44-1.00); Q1 cut-off) and

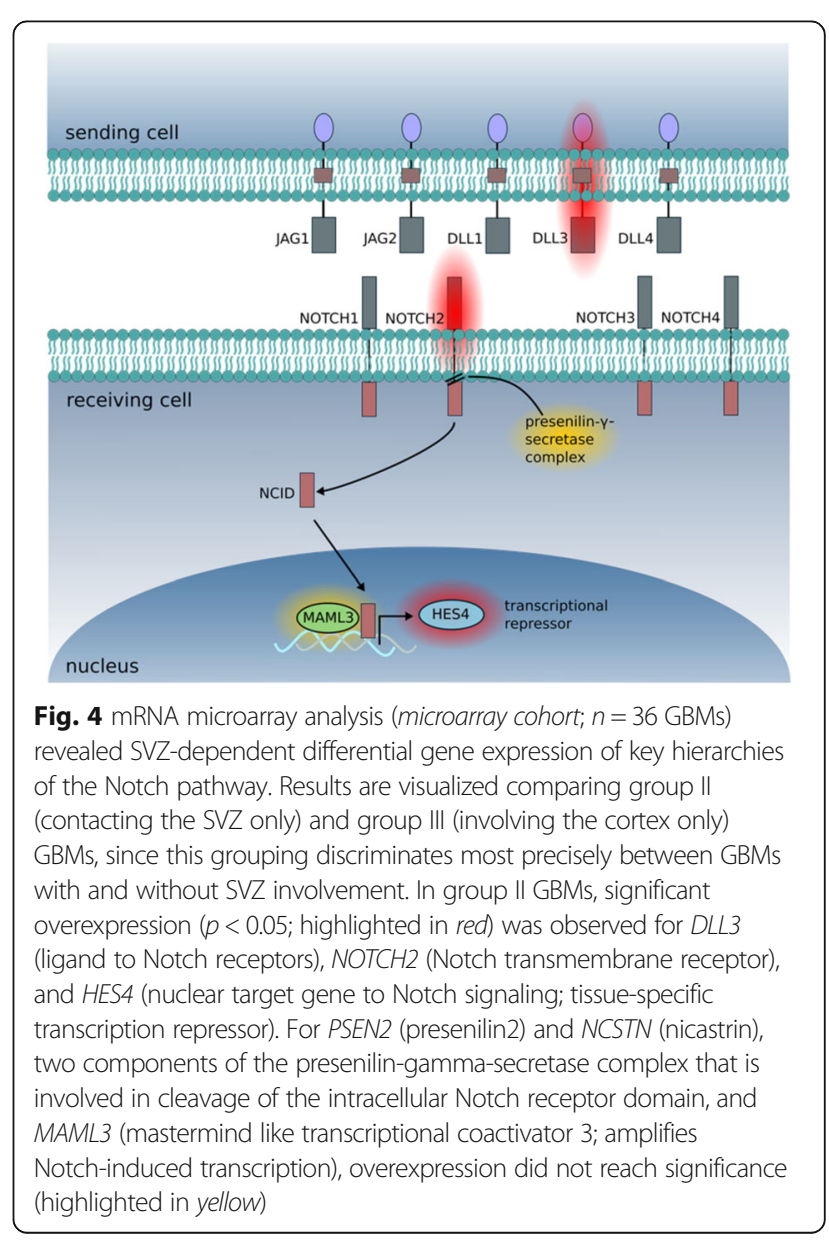


Table 5 Clinical and molecular prognostic factors of overall (OS) and progression-free (PFS) survival in the validation cohort ( $n=142$ IDH wt patients) based on a univariate log-rank test and a multivariate Cox regression model

\begin{tabular}{|c|c|c|c|c|c|}
\hline & & \multicolumn{2}{|l|}{ OS } & \multicolumn{2}{|l|}{ PFS } \\
\hline & & HR $(95 \% \mathrm{Cl})$ & $p$ value & HR $(95 \%$ Cl) & $p$ value \\
\hline \multicolumn{6}{|l|}{ Univariate } \\
\hline \multicolumn{6}{|c|}{ Clinico-pathological parameters } \\
\hline Age at first diagnosis & & $1.05(1.03-1.07)$ & $4.0 \mathrm{E}-07$ & $1.02(1.00-1.04)$ & 0.020 \\
\hline Gender (male) & & $0.99(0.70-1.41)$ & 0.971 & $1.33(0.87-2.04)$ & 0.187 \\
\hline Radiotherapy & & $0.29(0.16-0.52)$ & 9.7E-06 & $0.99(0.31-3.13)$ & 0.983 \\
\hline Chemotherapy & & $0.41(0.28-0.59)$ & $1.4 \mathrm{E}-06$ & $0.58(0.37-0.91)$ & 0.017 \\
\hline Preoperative KPS & & $0.98(0.97-0.99)$ & 0.002 & $1.00(0.99-1.01)$ & 0.810 \\
\hline MGMT methylation & & $0.83(0.53-1.29)$ & 0.401 & $1.15(0.69-1.92)$ & 0.588 \\
\hline GTR & & $0.42(0.28-0.62)$ & $8.8 \mathrm{E}-06$ & $0.78(0.49-1.23)$ & 0.286 \\
\hline Location (SVZ+) & & 1.39 (0.99-1.96) & 0.056 & $1.07(0.71-1.61)$ & 0.743 \\
\hline Location (II vs. III) & & $1.19(0.69-2.08)$ & 0.526 & $1.30(0.66-2.60)$ & 0.445 \\
\hline \multicolumn{6}{|l|}{ Candidate genes } \\
\hline & Cut-offs & & & & \\
\hline IGFBP5 & Q1 & $1.62(1.09-2.39)$ & 0.015 & $1.19(0.73-1.94)$ & 0.475 \\
\hline DLL3 & Median & $1.61(1.08-2.39)$ & 0.017 & $1.64(1.01-2.68)$ & 0.043 \\
\hline NTRK2 & Q1 & $0.66(0.44-1.00)$ & 0.049 & $0.64(0.39-1.07)$ & 0.086 \\
\hline PIR & Q1 & $0.62(0.41-0.95)$ & 0.025 & $0.66(0.40-1.08)$ & 0.092 \\
\hline HES4 & Q3 & $1.55(1.03-2.32)$ & 0.034 & $1.31(0.81-2.13)$ & 0.268 \\
\hline \multicolumn{6}{|l|}{ Multivariate } \\
\hline \multicolumn{6}{|c|}{ Clinico-pathological parameters } \\
\hline Age at first diagnosis & & $1.03(1.00-1.06)$ & 0.024 & $1.01(0.99-1.04)$ & 0.198 \\
\hline Radiotherapy & & $0.22(0.08-0.58)$ & 0.002 & & \\
\hline Chemotherapy & & $0.55(0.29-1.05)$ & 0.072 & $0.62(0.36-1.06)$ & 0.082 \\
\hline Preoperative KPS & & $0.99(0.98-1.01)$ & 0.504 & & \\
\hline GTR & & $0.48(0.29-0.83)$ & 0.007 & & \\
\hline Location (SVZ+) & & $1.82(1.09-3.04)$ & 0.023 & & \\
\hline \multicolumn{6}{|l|}{ Candidate genes } \\
\hline & Cut-offs & & & & \\
\hline IGFBP5 & Q1 & $0.82(0.48-1.42)$ & 0.488 & & \\
\hline DLL3 & Median & $1.47(0.83-2.60)$ & 0.181 & $1.65(1.00-2.70)$ & 0.046 \\
\hline NTRK2 & Q1 & $0.75(0.41-1.38)$ & 0.358 & & \\
\hline PIR & Q1 & $0.75(0.41-1.36)$ & 0.344 & & \\
\hline HES4 & Q3 & $2.03(1.06-3.90)$ & 0.033 & & \\
\hline
\end{tabular}

Gene expression data were dichotomized according to the median in "high" and "low" expression ("median cut-off") and according to quartiles either in "top $25 \%$ expression" and "<75\% expression" ("Q1 cut-off") or "bottom $25 \%$ expression" and ">25\% expression" ("Q3 cut-off"). $p$ values $<0.05$ are displayed in bold letters $H R$ hazard ratio, $\mathrm{Cl}$ confidence interval, KPS Karnofsky performance score, MGMT $O^{6}$-methylguanine-DNA methytransferase, GTR gross total resection

PIR ( $p=0.025$, HR 0.62 (0.41-0.95); Q1 cut-off) predicted superior OS. High expression of $D L L 3$ was also predictive of inferior PFS $(p=0.043$, HR $1.64(1.01-2.68)$; median cut-off). See Table 5. Also, a negative prognostic impact on OS was revealed for IGFBP5 $(p=0.015$, HR 1.62 (1.09-2.39); Q1 cut-off), one of the candidate genes for which location-dependent gene expression could not be confirmed in the validation cohort. Note that
NTRK2 and DLL3 were identified as location-specific prognostic markers: in SVZ+ GBMs, OS was significantly prolonged in patients with high NTRK2 expression levels ( $p=0.006$; Q1 cut-off; 18.5 versus 10.5 months). In group II GBMs, a significantly higher OS was observed in patients with high NTRK2 expression ( $p=0.042$; Q1 cutoff; 23 versus 8.5 months) and with low DLL3 expression ( $p=0.046$; median cut-off; 24 versus 13 months), 
respectively (Fig. 3c). Most importantly, multivariate survival analysis revealed a negative prognostic impact of HES4 on OS ( $p=0.033$; HR 2.03 (1.06-3.9)), independent of all other candidate genes and clinical factors with significant survival impact in univariate analysis, and of DLL3 on PFS $(p=0.046 ; \mathrm{HR} 1.65$ (1.00-2.70)). See Table 5.

Taken together, our stepwise approach identified four genes (HES4, DLL3, PIR, NTRK2) with SVZ-specific expression and simultaneous prognostic significance (summarized in Fig. 5). In multivariate survival analysis, HES4 was an independent prognosticator of OS and DLL3 of PFS. Both overall pathway analysis and in-depth analysis of single candidate genes point to a relevant involvement of Notch signaling in SVZ+ GBM.

\section{Discussion}

Intertumoral heterogeneity is one of the mainstays of treatment failure in GMB; thus, there is a need for individualized prognostication and treatment approaches. Tumor location is one important aspect that clearly determines treatment options, functional outcome, and quality of life. However, it is largely unknown whether tumor location is linked to a distinct molecular phenotype. In this study we sought to compare transcriptomes of GBMs with and without spatial relationship to the SVZ in order to identify location-dependent gene signatures and prognostic markers. In contrast to previous studies, locationdependent candidate genes identified in a discovery set were validated in an independent patient cohort comprising exclusively $I D H w t$ GBM. Thereby, we sought to exclude the unique molecular and prognostic phenotype of IDH $m t$ GBM [9].

Hierarchical clustering of microarray data revealed two main transcriptional profiles that perfectly matched the allocation of tumors according to their vicinity to

DIFFERENTIAL EXPRESSION
overaLL SURVIVAL
(prolonged)

the SVZ in general and to group II (GBMs contacting the SVZ only) and group III (GBMs contacting the cortex only) tumors in particular. MetaCore ${ }^{\text {max }}$ enrichment analysis linked these SVZ-dependent transcriptomes to major pathways involved in cell growth and motility, angiogenesis, immune modulation, and Notch signaling, one of the major developmental pathways involved in neural stem cell (NSC) maintenance and gliomagenesis $[26,27]$. Importantly, no overlap was found between our location-specific transcriptional profiles and the four molecular subgroups described by the TCGA [8]. In face of our study's relatively small case number, tumor vicinity to the SVZ does not appear as a determining factor of the TCGA's molecular phenotypes.

Ever since the early reports that vicinity of GBM to the SVZ is linked to a distinct growth behavior and inferior patient outcome [13-15], it has been hypothesized that SVZ+ GBM may arise from transformed NSCs originally residing within the SVZ [11, 12]. Indeed there is evidence from rodent studies that inactivation of typical tumor suppressor genes (TP53, NF1, PTEN) allocates formation and early progression of high-grade astrocytoma to neural stem/progenitor cells within the SVZ [28-30]. In humans, intraoperative fluorescence-guided multiple sampling (FGMS) along a spatial gradient within the tumor mass and the adjacent (fluorescent) SVZ recently allowed for a phylogenetic reconstruction with SVZ-derived tumor precursor cells giving rise to the tumor mass in the majority of GBM patients analyzed, and thus, for the first time, substantiated a role of the SVZ in gliomagenesis in humans [16]. These observations also suggest that SVZ+ GBMs are enriched in NSCs and BTICs, a hypothesis that has not yet been explored in much detail. In a microarray analysis of 47 GBMs, Kappadakunnel et al. did not find a correlation between 7 selected stem cell-related genes of interest (PROM1, MELK, BMP4, ETF2, MAPK8, OLIG2, NES) and tumor location [15]. Instead, 7 of the 33 genes overexpressed in group II tumors were related to immune signaling (FCGR3A, HLA-DRB5, BCL6, FCGR3B, MAFB, $H L A-D R A, H L A-E)$, a finding which was underscored by our MetaCore ${ }^{\text {tw }}$ pathway analysis.

To further investigate a potential stem cell origin of SVZ+ GMB, we searched our microarray data for location-specific differential expression of a panel of 47 well-known NSC, BTIC, and lineage markers as well as key players of three development pathways (Notch, Wnt, and Sonic Hedgehog). Since group I GBMs that are defined as SVZ+ GBMs consist of voluminous tumors that reach from the cortical surface to the SVZ and, in theory, may reflect tumors that originate from the cortex rather than the SVZ, we decided to compare group II and group III tumors only, since this reflects the most concise regional separation between GBMs with (group 
II) and without (group III) SVZ contact. Differential regulation of gene expression was observed for a subset of genes only (FZD6, PTCH2, NEFH, NOTCH2, HES4, DLL3), including three genes (NOTCH2, HES4, DLL3) involved in Notch signaling. Remarkably, locationdependent differential gene expression was detected at all key hierarchies of the Notch pathway (depicted in Fig. 4). It is well known that Notch signaling drives NSC maintenance and differentiation of neural progenitor cells into astroglia (reviewed in [27]). Moreover, aberrant Notch pathway activation contributes to formation and propagation of primary GBM [31], possibly through propagation of the BTIC pool [32], and therapeutic inhibition of Notch signaling is under investigation both in vitro and in vivo (reviewed in [33]). In this regard, our study provides the first link between Notch expression and tumor location. With the Notch ligand DLL3 and the transcription factor HES4 we found pivotal upregulated genes that later proved to be prognostic in a multivariate setting. However, our data are restricted to the transcriptional level, and functional analyses are needed to shed further light on location-specific Notch pathway activation. Nevertheless, neither this analysis nor that of Kappadakunnel et al. found unequivocal evidence of a (cancer) stem cell signature in SVZ+ GBM [15]. This may account for the fact that differential expression was assessed on a transcriptional level only and that in-depth analysis of a larger patient sample, in particular combined with investigation of protein expression, may yield unambiguous results. A sampling error may also come into play. Since all samples were retrospectively identified from our tumor bank, it is impossible to reconstruct the exact position of sampling, in particular with regard to distance from the SVZ. Particularly in group I GBM, the most voluminous location-specific subgroup extending from the SVZ throughout the white matter to cortical areas, there may well be a spatial gradient in the enrichment of tumor tissue with NSCs and BTICs, a factor impossible to take into account unless tissue sampling is done in a prospective manner with multiple samples derived from the same tumor and the regions of interest defined on perioperative imaging $[16,34]$. Ultimately, it would be worthwhile to compare transcriptomes from SVZ+ and SVZ- GBMs to those derived from non-malignant human SVZ to elucidate the role of the SVZ in human gliomagenesis. However, to the best of our knowledge, array data from human SVZ tissue have been lacking until now.

To attenuate the sampling issue, we ensured that all GBM tissues analyzed were homogeneous throughout the location groups in terms of tumor content and interplay from the microenvironment (Additional file 1: Figure S1A-D) and validated HES4 and DLL3 expression together with 11 other top differentially expressed candidate genes derived from microarray analysis in an independent set of $142 \mathrm{IDH} 1$ wild-type GBMs (validation cohort). Hence, we were able to confirm SVZdependent expression of HES4 and DLL3. HES4 was significantly overexpressed in both SVZ+ GBMs and group II GBMs. HES4 is one of seven HES target genes of Notch signaling that serve as tissue-specific transcription repressors upon Notch pathway activation, leading to inhibition of cell differentiation and maintenance of stem cell features [35]. Little is known about the particular function of HES4 in stem cells and cancer. HES4 was shown to keep retinal precursor cells of the Xenopus ciliary margin zone in an undifferentiated and slowly proliferative state [36]. In human B cells, HES4 inhibits early differentiation and acts as a tumor suppressor with epigenetic silencing in B cell acute lymphoblastic leukemia [37]. Recently, HES4 has been established as a biomarker in advanced solid tumors, predicting treatment response to Notch pathway inhibition by gammasecretase inhibitors $[38,39]$. Notably, our analysis constitutes the first report on HES4 interaction in human brain tumors.

$D L L 3$ is the second Notch pathway key player for which a strong trend towards overexpression in group II GBMs was confirmed in our analysis. DLL3 is a direct ligand to Notch receptors, with conflicting data about its activating or rather inhibiting role in Notch signaling [40-44]. Likewise, DLL3 has been described in the context of proneural GBM with inconsistent expression patterns: according to Phillips et al. [7] and Verhaak et al. [8], overexpression of $D L L 3$ is a hallmark of proneural GBM, while Cooper et al. [45] report loss of $D L L 3$ in proneural GBM. In the meantime, identification of an $I D H$-dependent G-CIMP phenotype has further separated the initially favorable appraised proneural molecular subgroup into GBMs with an $I D H$-mutant, GCIMP-positive phenotype exhibiting a highly favorable prognosis and an $I D H$-wild-type, non-G-CIMP phenotype with an exceedingly dismal prognosis [9]. This is of importance, since none of the aforementioned studies stratified expression and survival data for $I D H$ mutation status, while our study comprised IDH1 wt GBM only. Indeed, $D L L 3$ was significantly overexpressed in the 10 tumor samples of our microarray cohort assigned to the proneural subtype $(p=0.0078$; Mann-Whitney test; data not shown), but no difference in OS was observed between proneural and other subtypes $(p=0.4776$; logrank test; data not shown).

We also observed a strong trend towards upregulation of NTRK2 and downregulation of PIR in group II GBMs (contacting the SVZ only). NTRK2 encodes for the neurotrophic tyrosine kinase, receptor, type 2, also known as Trk-B. Neurotrophins and their receptors are crucial for cell growth, survival, and apoptosis in the 
nervous system [46], but expression has been observed in glioma as well [47], even though their functional role is largely unknown. Activation of Trk-B and Trk-C has been shown to promote growth and survival of BTICs independent of epidermal growth factor (EGF) and basic fibroblast growth factor (bFGF) [48]. In our study, NTRK2 overexpression was found in GBMs with SVZ contact, possibly maintaining BTIC growth as well. PIR is another location-specific candidate gene with downregulation observed in group II GBMs. It encodes for the iron-binding nuclear protein pirin, a transcriptional regulator, and has been described as an oncogene [49] and promoter of metastatic tumor growth [50] on one hand and as a tumor suppressor gene on the other [51] in many solid cancers, but never before in glioma. In acute myeloid leukemia (AML), PIR was linked to terminal differentiation of myeloid precursors with a downregulation of PIR possibly related to the differentiation arrest observed in AML [52]. By contrast, overexpression of PIR was involved in inhibition of cellular senescence in melanocytic cells, resulting in transformation to melanoma [53]. In GBM, the functional role of PIR has yet to be discovered.

Besides their SVZ-dependent expression, HES4, DLL3, $P I R$, and NTRK2 also conferred a significant impact on patient survival, together with known clinico-pathological prognosticators. Importantly, the reported adverse effect of SVZ involvement on OS [13] was confirmed in our study sample by multivariate analyses. In line with its distinct expression in GBMs contacting the SVZ, HES4 turned out to be the most robust prognostic marker with overexpression related to adverse OS, even overriding the prognostic effect of all other molecular markers in the multivariate analysis. As elucidated above, HES4 is a novel molecular marker in GBM and underscores the biological and clinical role of Notch pathway activation in primary GBM, in particular in tumors involving the SVZ. Likewise, overexpression of $D L L 3$, the second molecular marker involved in Notch signaling, resulted in significantly reduced OS and PFS in the univariate analysis and turned out to be an independent prognostic marker of inferior PFS in the multivariate analysis. Moreover, high expression of PIR and NTRK2 was associated with superior OS. It is noteworthy that in patients with GBMs contacting the SVZ (SVZ+ GBMs, group II GBMs), NTRK2 expression levels were able to predict OS. The beneficial effect of NTRK2 overexpression on OS confirms recent data reporting that loss of mRNA expression of both NTRK1 and NTRK2 correlates with poor prognosis in patients with high-grade glioma [54], but our analysis adds a location-specific link to the picture. To our knowledge, this is the first report on PIR as a prognostic marker in GBM, with evidence of SVZ-dependent differential expression, and it is worth further functional analysis to investigate its role as an oncogenic or tumor repressive factor. In addition to these four genes with SVZdependent differential expression and concurrent prognostic impact, we also identified IGFBP5 expression to be inversely related to OS, even though a location-specific expression could not be established. There is sparse evidence from the literature that overexpression of IGFBP5 increases in a WHO grade-dependent fashion with highest expression observed in GBM $[55,56]$ and a strong trend linking overexpression to adverse OS [55]. The functional role of IGFBP5 has not been fully clarified, but it may play a role in tumor dormancy, among others in GBM [57].

\section{Conclusions}

In summary, this study revealed inherent transcriptional differences of GBMs, depending on their vicinity to the SVZ. Interestingly, all four genes with simultaneous SVZ-dependent differential expression and significant prognostic impact were characterized by their involvement in stem cell maintenance. Two of these genes (HES4, PIR) have never before been reported in the context of gliomagenesis and deserve further functional exploration. Importantly, Notch signaling was an outstanding feature of SVZ+ GBM, with the two key players HES4 and DLL3 identified as location-specific prognosticators. Further work will be required, but this finding suggests that SVZ+ GBM might profit most from the therapeutic Notch inhibition that is currently under investigation in clinical trials. Mounting evidence is in favor of location-tailored therapies, since irradiation of the ipsilateral SVZ as a potential BTIC niche has been shown to have a positive impact on patient outcome [17, 18], particularly in patients with GTR [19]. SVZ-specific targeted molecular therapies might add another important piece to the picture.

\section{Additional files}

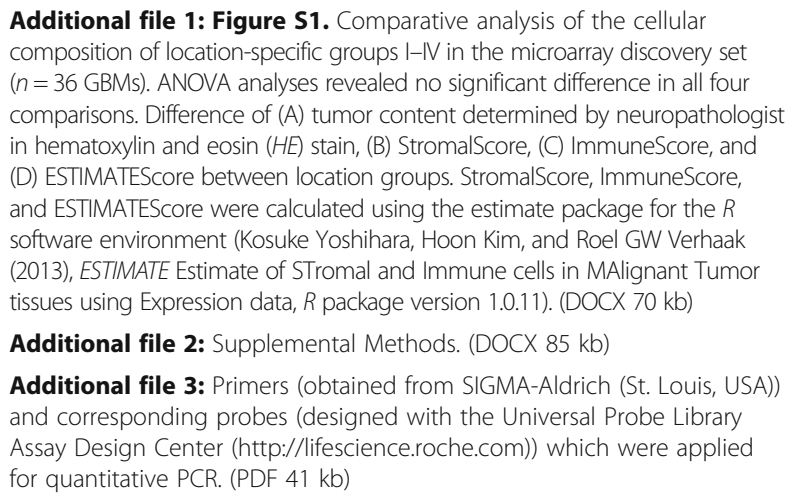

Additional file 2: Supplemental Methods. (DOCX $85 \mathrm{~kb}$ )

Additional file 3: Primers (obtained from SIGMA-Aldrich (St. Louis, USA)) and corresponding probes (designed with the Universal Probe Library Assay Design Center (http://lifescience.roche.com)) which were applied for quantitative PCR. (PDF $41 \mathrm{~kb}$ )

\section{Abbreviations}

AML: Acute myeloid leukemia; BTIC: Brain tumor-initiating cells; CE: Contrastenhancing; DLL3: Delta-like 3; EOR: Extent of resection; FGMS: Fluorescenceguided multiple sampling; GBM: Glioblastoma; G-CIMP: Glioma-CpG island methylator phenotype; GTR: Gross total resection; HES4: Hairy and enhancer 
of split 4; IDH1: Isocitrate dehydrogenase 1; IGFBP5: Insulin-like growth factor-binding protein 5; MGMT: $O^{6}$-methylguanine-DNA methyltransferase; mt: Mutant; NSC: Neural stem cell; NTRK2: Neurotrophic receptor tyrosine kinase, type 2; OS: Overall survival; PFS: Progression-free survival; PIR: Pirin; qPCR: Quantitative polymerase chain reaction; SVZ: Subventricular zone; TCGA: The Cancer Genome Atlas; TMZ: Temozolomide; WHO: World Health Organization; wt: Wild-type

\section{Acknowledgements}

We thank Daniela Zito, Anja Metzner, and Mandy Barthel for their review of patient data as well as Farzaneh Kashfi, Hildegard Göltzer, Ilka Hearn, and Melanie Greibich for their excellent technical support.

\section{Funding}

This work was supported by the "Bundesministerium für Bildung und Forschung" (01GS0886 to CHM) and a grant from the "Deutsche Krebshilfe" (German Cancer Aid; 109202 to CHM).

\section{Availability of data and materials}

Data were deposited at the NCBI Gene Expression Omnibus [GEO:GSE83537].

\section{Authors' contributions}

CJ conceived, designed, and supervised the study, undertook analysis and interpretation of data, and drafted the manuscript. AM conducted MetaCore ${ }^{\text {TM }}$ enrichment analyses as well as statistical analyses and interpretation of microarray and clinical data and critically revised the manuscript. JE performed qPCR analyses and helped with interpretation of data. CG undertook correlation of microarray data with the TCGA's molecular subtypes. RW designed and supervised the study-specific mRNA microarray. DC and AvD performed histopathological evaluation, quality control of tumor tissue, and confirmation of IDH1 and MGMT status. AA designed and supervised MetaCore ${ }^{\text {TM }}$ enrichment analyses. SF helped with tissue sampling and RNA extraction. BL and NG performed digitalization of tumor tissue slides. PB supervised the study-specific mRNA microarray. AU critically revised the manuscript. CHM supervised the study, undertook interpretation of data, and critically revised the manuscript. All authors read and approved the final manuscript.

\section{Competing interests}

The authors declare that they have no competing interests.

\section{Ethics approval and consent to participate}

Tissue sampling and molecular analysis as well as collection of clinical data were performed after obtaining written informed consent from patients in all cases, with approval from the ethics committee of the Ruprecht-Karls University Heidelberg (reference 005/2003).

\section{Author details}

'Division of Experimental Neurosurgery, Department of Neurosurgery, Ruprecht-Karls-University Heidelberg, Im Neuenheimer Feld 400, 69120 Heidelberg, Germany. ${ }^{2}$ Department of Neuropathology, Heidelberg University Hospital; CCU Neuropathology, German Consortium for Translational Cancer Research (DKTK), German Cancer Research Center (DKFZ), Heidelberg, Germany. ${ }^{3}$ Department of Radiation Oncology, Heidelberg University Hospital; Molecular and Translational Radiation Oncology, National Center for Tumor Diseases (NCT), German Cancer Research Center (DKFZ), Heidelberg, Germany. ${ }^{4}$ Hamamatsu Tissue and Imaging Analysis Center, University of Heidelberg, Heidelberg, Germany. ${ }^{5}$ Division of Translational Immunology, German Cancer Research Center (DKFZ), Heidelberg, Germany. ${ }^{6}$ Regensburg Center for Interventional Immunology (RCI), University Hospital, Regensburg, Germany.

\section{Received: 16 July 2016 Accepted: 1 October 2016}

\section{Published online: 26 October 2016}

\section{References}

1. Stupp R, Mason WP, van den Bent MJ, Weller M, Fisher B, Taphoorn MJB, et al. Radiotherapy plus concomitant and adjuvant temozolomide for glioblastoma. N Engl J Med. 2005;352(10):987-96.

2. Krex D, Klink B, Hartmann C, von Deimling A, Pietsch T, Simon M, et al. Long-term survival with glioblastoma multiforme. Brain J Neurol. 2007; 130(Pt 10):2596-606.
3. Stummer W, Pichlmeier U, Meinel T, Wiestler OD, Zanella F, Reulen $\mathrm{H}-\mathrm{J}$, et al. Fluorescence-guided surgery with 5-aminolevulinic acid for resection of malignant glioma: a randomised controlled multicentre phase III trial. Lancet Oncol. 2006;7(5):392-401.

4. Stummer W, Reulen H-J, Meinel T, Pichlmeier U, Schumacher W, Tonn J-C, et al. Extent of resection and survival in glioblastoma multiforme: identification of and adjustment for bias. Neurosurgery. 2008;62(3):564-76.

5. Hottinger AF, Stupp R, Homicsko K. Standards of care and novel approaches in the management of glioblastoma multiforme. Chin J Cancer. 2014;33(1):32-9.

6. Patel MA, Kim JE, Ruzevick J, Li G, Lim M. The future of glioblastoma therapy: synergism of standard of care and immunotherapy. Cancers. 2014;6(4):1953-85.

7. Phillips HS, Kharbanda S, Chen R, Forrest WF, Soriano RH, Wu TD, et al. Molecular subclasses of high-grade glioma predict prognosis, delineate a pattern of disease progression, and resemble stages in neurogenesis. Cancer Cell. 2006;9(3):157-73.

8. Verhaak RGW, Hoadley KA, Purdom E, Wang V, Qi Y, Wilkerson MD, et al. Integrated genomic analysis identifies clinically relevant subtypes of glioblastoma characterized by abnormalities in PDGFRA, IDH1, EGFR, and NF1. Cancer Cell. 2010;17(1):98-110.

9. Noushmehr H, Weisenberger DJ, Diefes K, Phillips HS, Pujara K, Berman BP, et al. Identification of a CpG island methylator phenotype that defines a distinct subgroup of glioma. Cancer Cell. 2010;17(5):510-22.

10. Turcan S, Rohle D, Goenka A, Walsh LA, Fang F, Yilmaz E, et al. IDH1 mutation is sufficient to establish the glioma hypermethylator phenotype. Nature. 2012;483(7390):479-83.

11. Sanai N, Tramontin AD, Quiñones-Hinojosa A, Barbaro NM, Gupta N, Kunwar S, et al. Unique astrocyte ribbon in adult human brain contains neural stem cells but lacks chain migration. Nature. 2004;427(6976):740-4.

12. Sanai N, Alvarez-Buylla A, Berger MS. Neural stem cells and the origin of gliomas. N Engl J Med. 2005;353(8):811-22.

13. Jafri NF, Clarke JL, Weinberg V, Barani IJ, Cha S. Relationship of glioblastoma multiforme to the subventricular zone is associated with survival. Neuro Oncol. 2013;15(1):91-6.

14. Lim DA, Cha S, Mayo MC, Chen M-H, Keles E, VandenBerg S, et al. Relationship of glioblastoma multiforme to neural stem cell regions predicts invasive and multifocal tumor phenotype. Neuro Oncol. 2007:9(4):424-9.

15. Kappadakunnel M, Eskin A, Dong J, Nelson SF, Mischel PS, Liau LM, et al. Stem cell associated gene expression in glioblastoma multiforme: relationship to survival and the subventricular zone. J Neurooncol. 2010;96(3):359-67.

16. Piccirillo SGM, Spiteri I, Sottoriva A, Touloumis A, Ber S, Price SJ, et al. Contributions to drug resistance in glioblastoma derived from malignant cells in the sub-ependymal zone. Cancer Res. 2015;75(1):194-202.

17. Gupta T, Nair V, Paul SN, Kannan S, Moiyadi A, Epari S, et al. Can irradiation of potential cancer stem-cell niche in the subventricular zone influence survival in patients with newly diagnosed glioblastoma? J Neurooncol. 2012;109(1):195-203.

18. Lee P, Eppinga W, Lagerwaard F, Cloughesy T, Slotman B, Nghiemphu PL, et al. Evaluation of high ipsilateral subventricular zone radiation therapy dose in glioblastoma: a pooled analysis. Int J Radiat Oncol Biol Phys. 2013;86(4):609-15.

19. Chen L, Guerrero-Cazares H, Ye X, Ford E, McNutt T, Kleinberg L, et al. Increased subventricular zone radiation dose correlates with survival in glioblastoma patients after gross total resection. Int J Radiat Oncol Biol Phys. 2013;86(4):616-22.

20. Yoshihara K, Shahmoradgoli M, Martínez E, Vegesna R, Kim H, Torres-Garcia $W$, et al. Inferring tumour purity and stromal and immune cell admixture from expression data. Nat Commun. 2013;4:2612.

21. Capper D, Weissert S, Balss J, Habel A, Meyer J, Jäger D, et al. Characterization of $\mathrm{R} 132 \mathrm{H}$ mutation-specific IDH1 antibody binding in brain tumors. Brain Pathol Zurich Switz. 2010;20(1):245-54.

22. Hartmann C, Meyer J, Balss J, Capper D, Mueller W, Christians A, et al. Type and frequency of $\mathrm{IDH} 1$ and $\mathrm{IDH} 2$ mutations are related to astrocytic and oligodendroglial differentiation and age: a study of 1,010 diffuse gliomas. Acta NeuropAthol (Berl). 2009;118(4):469-74.

23. Eberwine J, Yeh H, Miyashiro K, Cao Y, Nair S, Finnell R, et al. Analysis of gene expression in single live neurons. Proc Natl Acad Sci U S A. 1992;89(7):3010-4.

24. Gautier L, Cope L, Bolstad BM, Irizarry RA. affy — analysis of Affymetrix GeneChip data at the probe level. Bioinforma Oxf Engl. 2004;20(3):307-15.

25. Workman $C$, Jensen $\sqcup$, Jarmer H, Berka R, Gautier L, Nielser HB, et al. A new non-linear normalization method for reducing variability in DNA microarray experiments. Genome Biol. 2002;3(9):research0048.

26. Pierfelice TJ, Schreck KC, Eberhart CG, Gaiano N. Notch, neural stem cells, and brain tumors. Cold Spring Harb Symp Quant Biol. 2008;73:367-75. 
27. Teodorczyk M, Schmidt MHH. Notching on cancer's door: notch signaling in brain tumors. Front Oncol. 2014;4:341.

28. Alcantara Llaguno S, Chen J, Kwon C-H, Jackson EL, Li Y, Burns DK, et al. Malignant astrocytomas originate from neural stem/progenitor cells in a somatic tumor suppressor mouse model. Cancer Cell. 2009;15(1):45-56.

29. Kwon C-H, Zhao D, Chen J, Alcantara S, Li Y, Burns DK, et al. Pten haploinsufficiency accelerates formation of high-grade astrocytomas. Cancer Res. 2008;68(9):3286-94.

30. Zhu Y, Guignard F, Zhao D, Liu L, Burns DK, Mason RP, et al. Early inactivation of p53 tumor suppressor gene cooperating with NF1 loss induces malignant astrocytoma. Cancer Cell. 2005;8(2):119-30.

31. Purow BW, Haque RM, Noel MW, Su Q, Burdick MJ, Lee J, et al. Expression of Notch-1 and its ligands, Delta-like-1 and Jagged-1, is critical for glioma cell survival and proliferation. Cancer Res. 2005;65(6):2353-63.

32. Fan X, Khaki L, Zhu TS, Soules ME, Talsma CE, Gul N, et al. NOTCH pathway blockade depletes CD133-positive glioblastoma cells and inhibits growth of tumor neurospheres and xenografts. Stem Cells Dayt Ohio. 2010;28(1):5-16.

33. Stockhausen M-T, Kristoffersen K, Poulsen HS. The functional role of Notch signaling in human gliomas. Neuro Oncol. 2010;12(2):199-211.

34. Sottoriva A, Spiteri I, Piccirillo SGM, Touloumis A, Collins VP, Marioni JC, et al. Intratumor heterogeneity in human glioblastoma reflects cancer evolutionary dynamics. Proc Natl Acad Sci U S A. 2013;110(10):4009-14.

35. Kageyama R, Ohtsuka T, Kobayashi T. Roles of Hes genes in neural development. Dev Growth Differ. 2008;50 Suppl 1:S97-S103.

36. El Yakoubi W, Borday C, Hamdache J, Parain K, Tran HT, Vleminckx K, et al. Hes4 controls proliferative properties of neural stem cells during retinal ontogenesis. Stem Cells Dayt Ohio. 2012;30(12):2784-95.

37. Kuang S-Q, Fang Z, Zweidler-McKay PA, Yang H, Wei Y, Gonzalez-Cervantes EA, et al. Epigenetic inactivation of Notch-Hes pathway in human B-cell acute lymphoblastic leukemia. PLoS One. 2013;8(4):e61807.

38. Stoeck A, Lejnine S, Truong A, Pan L, Wang H, Zang C, et al. Discovery of biomarkers predictive of GSI response in triple-negative breast cancer and adenoid cystic carcinoma. Cancer Discov. 2014;4(10):1154-67.

39. Messersmith WA, Shapiro Gl, Cleary JM, Jimeno A, Dasari A, Huang B, et al. A Phase I, dose-finding study in patients with advanced solid malignancies of the oral $y$-secretase inhibitor PF-03084014. Clin Cancer Res. 2015;21(1):60-7.

40. Ladi E, Nichols JT, Ge W, Miyamoto A, Yao C, Yang L-T, et al. The divergent DSL ligand DII3 does not activate Notch signaling but cell autonomously attenuates signaling induced by other DSL ligands. J Cell Biol. 2005;170(6):983-92.

41. Tso C-L, Shintaku P, Chen J, Liu Q, Liu J, Chen Z, et al. Primary glioblastomas express mesenchymal stem-like properties. Mol Cancer Res. 2006;4(9):607-19.

42. Geffers I, Serth K, Chapman G, Jaekel R, Schuster-Gossler K, Cordes R, et al. Divergent functions and distinct localization of the Notch ligands DLL1 and DLL3 in vivo. J Cell Biol. 2007:178(3):465-76.

43. Hu B, Nandhu MS, Sim H, Agudelo-Garcia PA, Saldivar JC, Dolan CE, et al. Fibulin-3 promotes glioma growth and resistance through a novel paracrine regulation of Notch signaling. Cancer Res. 2012;72(15):3873-85.

44. Turchi L, Debruyne DN, Almairac F, Virolle $V$, Fareh M, Neirijnck Y, et al. Tumorigenic potential of miR-18A* in glioma initiating cells requires NOTCH-1 signaling. Stem Cells Dayt Ohio. 2013;31(7):1252-65.

45. Cooper LAD, Gutman DA, Long Q, Johnson BA, Cholleti SR, Kurc T, et al. The proneural molecular signature is enriched in oligodendrogliomas and predicts improved survival among diffuse gliomas. PLoS One. 2010;5(9):e12548.

46. Chao MV. Neurotrophins and their receptors: a convergence point for many signalling pathways. Nat Rev Neurosci. 2003;4(4):299-309.

47. Assimakopoulou M, Kondyli M, Gatzounis G, Maraziotis T, Varakis J. Neurotrophin receptors expression and JNK pathway activation in human astrocytomas. BMC Cancer. 2007;7:202.

48. Lawn S, Krishna N, Pisklakova A, Qu X, Fenstermacher DA, Fournier M, et al. Neurotrophin signaling via TrkB and TrkC receptors promotes the growth of brain tumor-initiating cells. J Biol Chem. 2015;290(6):3814-24.

49. Bergman AC, Alaiya AA, Wendler W, Binetruy B, Shoshan M, Sakaguchi K, et al. Protein kinase-dependent overexpression of the nuclear protein pirin in C-JUN and RAS transformed fibroblasts. Cell Mol Life Sc. 1999;55(3):467-71.

50. Shubbar E, Helou K, Kovács A, Nemes S, Hajizadeh S, Enerbäck C, et al. High levels of $\gamma$-glutamyl hydrolase $(\mathrm{GGH})$ are associated with poor prognosis and unfavorable clinical outcomes in invasive breast cancer. BMC Cancer. 2013;13:47.

51. Qiao Z, Wang D, Hahn J, Ai J, Wang Z. Pirin down-regulates the EAF2/U19 protein and alleviates its growth inhibition in prostate cancer cells. Prostate. 2014;74(2):113-20.
52. Licciulli S, Cambiaghi V, Scafetta G, Gruszka AM, Alcalay M. Pirin downregulation is a feature of AML and leads to impairment of terminal myeloid differentiation. Leukemia. 2010;24(2):429-37.

53. Licciulli S, Luise C, Scafetta G, Capra M, Giardina G, Nuciforo P, et al. Pirin inhibits cellular senescence in melanocytic cells. Am J Pathol. 2011;178(5):2397-406.

54. Palani M, Arunkumar R, Vanisree AJ. Methylation and expression patterns of tropomyosin-related kinase genes in different grades of glioma. Neuromolecular Med. 2014;16(3):529-39.

55. Santosh V, Arivazhagan A, Sreekanthreddy P, Srinivasan H, Thota B, Srividya MR, et al. Grade-specific expression of insulin-like growth factor-binding proteins-2, -3, and -5 in astrocytomas: IGFBP-3 emerges as a strong predictor of survival in patients with newly diagnosed glioblastoma. Cancer Epidemiol Biomark Prev. 2010;19(6):1399-408.

56. Wang H, Wang H, Zhang W, Fuller GN. Overexpression of IGFBP5, but not IGFBP3, correlates with the histologic grade of human diffuse glioma: a tissue microarray and immunohistochemical study. Technol Cancer Res Treat. 2006;5(3):195-9.

57. Satchi-Fainaro R, Ferber S, Segal E, Ma L, Dixit N, ljaz A, et al. Prospective identification of glioblastoma cells generating dormant tumors. PLoS One. 2012;7(9):e44395.

\section{Submit your next manuscript to BioMed Central and we will help you at every step:}

- We accept pre-submission inquiries

- Our selector tool helps you to find the most relevant journal

- We provide round the clock customer support

- Convenient online submission

- Thorough peer review

- Inclusion in PubMed and all major indexing services

- Maximum visibility for your research

Submit your manuscript at www.biomedcentral.com/submit
C Biomed Central 